\title{
Perinatal Transmission of HIV and Syphilis in Mexico
}

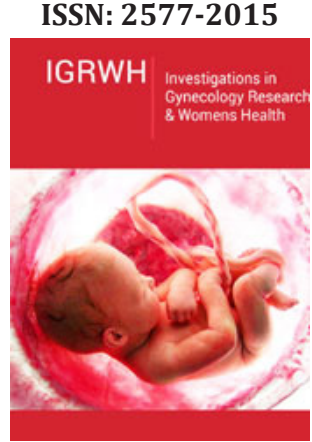

*Corresponding author: Posadas Robledo FJ, Department of Health Services of San Luis Potosí, México

Submission: 毕June 14, 2019

Published: 觜July 09, 2018

Volume 3 - Issue 1

How to cite this article: Posadas Robledo FJ. Perinatal Transmission of HIV and Syphilis in Mexico. Invest Gynecol Res Women's Health.3(1). IGRWH.000554.2019.

DOI: 10.31031/IGRWH.2019.03.000554

Copyright@ Posadas Robledo FJ, This article is distributed under the terms of the Creative Commons Attribution 4.0 International License, which permits unrestricted use and redistribution provided that the original author and source are credited.

\author{
Posadas Robledo FJ* \\ Department of Health Services of San Luis Potosí, México
}

\section{Mini Review}

In the international context, HIV and AIDS are a clear and specific sphere of attention, in which Mexico has sustained the commitment to adhere to global agreements that aim to respond to this epidemic, as well as to respect human rights, to combat the stigma and discrimination suffered by people who live and coexist with the virus and the infection. The essential objectives of the national response include the control of perinatal transmission of HIV and syphilis. These indicators are very sensitive and directly reflect the discrimination that exists towards the attention of women and their health problems. The process is complicated by unresolved social determinants. Poverty, low educational level and limited access to quality health services are the main factors that prevent optimal control of these infections. In addition, the disarticulation of a fragmented health system and the significant number of births, exceeding 2.2 million each year, are added.

The essential goal is to eliminate vertical transmission of HIV and congenital syphilis to achieve the first free generation of these infections by 2030. Intermediate goals include achieving prenatal screening coverage for the HIV and syphilis binomial of 95\%. Treatment and control of $90 \%$ of reactive pregnant women and their newborns. The main clinical strategy for pregnant women is to screen for them. Once they are detected with HIV. The diagnosis should be confirmed, and treatment should be administered after 16 weeks of gestation, or after diagnosis and lead to suppressed or undetectable viral load and timely interruption of pregnancy and avoid breastfeeding to limit the risk of perinatal transmission. The neonate should be screened at birth in addition to tests for virologic diagnosis and keep monitoring for a period of 2 to 5 years. The management should be supplemented with sieve for syphilis and, if reactive, administer antibiotic treatment early to avoid neonatal sepsis, death or stillbirth.

In Mexico, for the purpose of preventing perinatal transmission of HIV, the follow-up of reactive pregnant women tested for HIV and / or syphilis is nominal. At the end of 2018, 81 new cases of HIV were registered through vertical transmission, the main gap that has not been closed is the low coverage of HIV detection in pregnant women. Of the new cases in this last year, $86 \%$ are concentrated in 17 states of the country. The goal is to achieve less than 20 cases per year. Countries must achieve a transmission rate of less than $2 \%$ in population groups that do not practice breastfeeding. Also, according to preliminary figures 2018, 252 new cases of congenital syphilis were reported, $70 \%$ of which were detected in 6 states. For syphilis, the goal is to achieve less than 50 cases of congenital syphilis per 100,000 live births. In Mexico, less than 20 cases per year.

In Mexico, approximately 13,000 fetal deaths are recorded each year. A national strategy is in the process of implementing screening for syphilis in deaths and stillbirths and verifying how many cases can be caused by this infection, which as we know is feticide when acquired in the early stages of pregnancy, before 20 weeks and reduce the risk of under-registration of the infection [1,2]. The National Center for the Prevention and Control of HIV and AIDS (Censida). It depends on the Ministry of Health and has the faculty to conduct the public policies of the sector and its main activity is to play a leading role to provide services in the national response to HIV and other Sexually Transmitted Infections (ITS's). In the preventive, curative and at all levels of care. 


\section{Challenges}

In the month of December 2018, Mexico began a profound process of political and social change. One of the priorities of the new regime is to consolidate a health system in accordance with social demands, which allows health indicators to be impacted yet to be resolved. HIV and perinatal syphilis are two very sensitive indicators of social justice, which is why they become a national priority in public policies. To achieve the expected impact, there is the enormous challenge of consolidating a health system with universal access, financed by general taxes, with homogeneous quality standards, in addition to focusing on users and having the person in the center of the system. Primary care is essential, which allows exercising preventive medicine as a basis for the new social model. Providing comprehensive quality care, to keep people healthy, productive and with the ability to seek their own satisfaction, is the true national challenge, health is a prerequisite to achieve impacts and better social capital [3].

In terms of HIV and STIs, perinatal, the challenge is to increase the quality of obstetric and perinatal care and achieve definitive control of new cases of HIV and STIs in pregnant women, through prevention, diagnosis and timely treatment. Discrimination and stigma remain a social problem that impacts health and other indicators of social well-being. The new system of government is promoting a culture of respect for diversity, human rights and interculturality. That allow in a short time to consolidate a culture of social inclusion and respect for diversity in all its manifestations. It is important to increase the quality and opportunity of contraceptive counseling for people and couples with HIV. At the end of the road, you find the essential goal as a country; achieve eliminate the vertical transmission of HIV and congenital syphilis, to achieve the first generation free of these infections. It is an important step to close the gap in equal rights.

\section{References}

1. (2015) Global guidelines on the criteria and processes for the validation of elimination of mother-to-child transmission of HIV and syphilis. Global Health Organization pp. 1-24.

2. World Health Organization (2008) Global elimination of congenital syphilis: fundamentals and strategy for action. World Health Organization, Geneva, Switzerland, pp. 1-41.

3. Mayer Serra CE (2007) The right to have health protection. Salud Publica Mex 49(2): 144-155. 